\title{
Pulmonary edema lead by circulation overload following shoulder arthroscopy under general anesthesia with nerve block: an observational study
}

\section{Gang Zhang}

Sichuan Provincial Orthopedic Hospital

Qihai Wan

Chengdu University of Traditional Chinese Medicine

\section{Xiaoyan Huang}

Chengdu Third People's Hospital

Chunqiong Luo

Sichuan Provincial Orthopedic Hospital

Yunhua Shui

Sichuan Provincial Orthopedic Hospital

Lan Zhang ( $\nabla$ mzzhanggang@hotmail.com )

Sichuan Provincial Orthopedic Hospital

Research article

Keywords: Shoulder arthroscopy, Pulmonary edema, Ultrasound, B lines

Posted Date: March 21st, 2020

DOI: https://doi.org/10.21203/rs.3.rs-18195/v1

License: () (1) This work is licensed under a Creative Commons Attribution 4.0 International License.

Read Full License 


\section{Abstract}

Purpose : Shoulder arthroscopy requires a large of irrigation for a better surgical view, resulting in circulatory overload. This study was performed to $\mathrm{p}$ rove whether pulmonary edema will be lead by a large of irrigation .

Materials and Methods: General anesthesia with interscalene block was induced before operation. The primary outcome was ultrasound evaluation of $B$ lines from the time before nerve block to the time 10 hours after operation. The secondary outcomes included oxygenation index, arterial partial pressure of carbon dioxide, visual analogue scale, muscle strength grade.

Results : A total of 93 patients were evaluated. Before surgery, B lines failed to be detected. While the highest total incidence of $B$ lines was $49.4 \%$, occured at 4 hours after surgery. The highest incidence s of severe and moderate pulmonary edema were 3.2\% $(P=0.081)$ and $9.7 \%(P=0.002)$, respectively. $B$ lines were also found on both the affected and healthy side. During operation, the incidence of type 1 respiratory failure was $5.4 \%(P=0.023)$ and that of both type 1 and 2 respiratory failure were $6.5 \%$ $(P=0.013)$. Pain was relieved in 6 hours after surgery (VAS $<3)$. At 12 hours after operation, the VAS of resting and motion were $4.68 \pm 2.27,6.90 \pm 2.43$, respectively. While the grade of muscle strength was $4.48 \pm 0.51$ at 12 hours after operation.

Conclusions: There is a high incidence of pulmonary edema in shoulder arthroscopy, and ultrasound is an convenient tool to evaluate this complication. Pain is relieved in 6 hours after surgery by nerve block. While muscle strength can also recover at 12 hours after surgery.

\section{Background}

Shoulder arthroscopy, a minimally invasive surgery procedure, has become a routine way to diagnose and treat a variety of shoulder joint diseases compared with traditional surgery, it has obvious advantages such as a faster postoperative recovery and shorter hospital stay [1]. Despite these benefits it still has some shortcomings. For a better surgical view, it requires a large of irrigation fluid to distend the joint cavity, which may lead a serious extravasation to the adjacent soft tissues. Some surgery may damage shoulder capsule, leading the irrigation to permeate into the outer space. As a result, it may lead the occurrence of significant edema of face, neck and chest tissues, tracheal compression, upper respiratory tract obstruction, and pulmonary edema. For swelling of adjacent neck, chest, and facial tissues, most patients will be lack of obvious syndrome within 2 days after surgery[2]. However, in severe cases, excessive irrigation will flow into the pharyngolaryngeal and paratracheal space, which can lead upper respiratory obstruction $[3,4]$ and even pulmonary edema [5].

Complications caused by irrigation have been paid more and more attention [6]. Borgeat reported a case of life-threatening respiratory distress by the large extravasation [7]. Gogia reported a case of airway obstruction for severe neck edema after extubation, and then negative pressure pulmonary edema occured [5]. In this case, negative-pressure pulmonary edema is directly caused by airway obstruction. 
However, there is not any report of pulmonary edema caused by overload of circulation in shoulder arthroscopy. This complication caused by excessive flushing fluid has been reported in transurethral prostatectomy [8] and hysteroscopic surgery [9]. To ensure the surgical vision, shoulder arthroscopy also requires a large amount of irrigation, which may lead an overload of circulation. Structures near the shoulder joint is attached by a large of muscles rich of blood, which is the anatomic basis for absorption into the blood. Will the life-threatening pulmonary edema be lead by a large of irrigation fluid in shoulder arthroscopy? Ultrasound is a reliable technique for monitoring various pulmonary edema. Compared with $X$-ray, it has the advantages of no radiation and real-time [10]. The number of $B$ lines $\left(N_{B}\right)$ from ultrasound also plays important roles in evaluating severity of pulmonary edema [11]. In our hospital, swelling of adjacent tissues and pulmonary edema, have been observed in a number of patients by ultrasound. For ensuring safety, will the ultrasound be an convenient tool to rapidly assess and identify this complication? Consequently, to answer the two questions above, we performed this observational study.

\section{Methods}

This study was performed after approval by Research Ethics Committee of Sichuan Provincial Orthopedic Hospital (Chengdu Sports Hospital and Chengdu Research Institute for Sports Injury). Written informed consent was obtained from all the patients before study. It was also registed prior to patient enrollment at Chinese Clinical Trial Registry (http://www.chictr.org.cn; Registration number: ChiCTR1900023793; Principal investigator: Lan Zhang; Date of registration: June 12, 2019). This study adheres to the applicable CONSORT guidelines. Exclusion criteria included pulmonary diseases (chronic pulmonary diseases or pulmonary edema), body mass index $\geq 35 \mathrm{~kg} / \mathrm{m}^{2}$, unable to perform brachial plexus nerve block (skin infection, brachial plexus nerve injury), severe liver or kidney disease, abnormal coagulation function. Oxygen saturation, electrocardiogram, noninvasive blood pressure and body temperature were monitored once the patients had been into the operating room. Interscalene brachial plexus block (ISBPB) was performed guided by ultrasound and nerve stimulator before general anesthesia. Local anesthetics ( $0.33 \%$ ropivacaine, $30 \mathrm{ml})$ were also injected at the back of brachial plexus to provide adequate analgesia and muscle relaxation. Then the radial artery of healthy side was punctured and catheterized to monitor invasive blood pressure. Patients were preoxygenated and anesthesia were induced with sufentanil $0.5 \mathrm{ug} / \mathrm{kg}$ and propofol $2.5 \mathrm{mg} / \mathrm{kg}$. Neuromuscular blockades were followed by a single infusion of rocuronium $0.6 \mathrm{mg} / \mathrm{kg}$. Intubation was induced and the tidal volume of mechanical ventilation was set to $8-10 \mathrm{ml} / \mathrm{kg}$. Patients undergoing shoulder arthroscopy would be evaluated dynamically by pulmonary ultrasouond (Navi U, Wisonic Inc, $\mathrm{CN}$ ) from the begining of operation to the time at 10 hours after operation. Monitoring time points included the time before nerve block, the time after surgery (or time in postanesthesia care unit, PACU) and the time at 2, 4, 6, 8, 10 hours after operation $\left(T_{\text {be }}, T_{\text {pa }}, T_{2}, T_{4}, T_{6}, T_{8}, T_{10}\right)$. The second, third, fourth and fifth intercostal spaces of the right lung were scanned with convex array probe along the parasternal line, midclavicular line, anterior axillary line and midaxillary line, while the second, third and fourth intercostal spaces of the left lung were scanned along the same anatomic location. 
Fluid therapy was performed according to body weight. The normal physiological requirement was $4 \mathrm{ml} / \mathrm{kg} . \mathrm{h}$ for the first $10 \mathrm{~kg}, 2 \mathrm{ml} / \mathrm{kg}$.h for the second $10 \mathrm{~kg}$, and $1 \mathrm{ml} / \mathrm{kg}$.h for remaining weight (RW). The formula to calculate the volume of fluid therapy was listed below $\left(T_{f+o}\right.$ : Time for fasting and operation).

Volume $(\mathrm{ml})=[10 \times 4+10 \times 2+R W(\mathrm{~kg}) \times 1] \times \mathrm{T}_{\mathrm{f+o}}(\mathrm{h})$

The primary outcome was continuous number counting of B lines in 10 hours after surgery. B lines from ultrasound were also used as an important diagnostic indicator of pulmonary edema. According to $N_{B}$, patients were classified as 4 groups (non-pulmonary edema: $N B \leq 5$; mild pulmonary edema: $N B=6-15$; moderate pulmonary edema: $N B=16-29$; severe pulmonary edema: $N B \geq 30)^{11}$. While respiratory parameters were also monitored by blood gas analysis (BGA). Oxygenation index (OI) was equal to the ratio of partial arterial $\mathrm{O}_{2}$ pressure $\left(\mathrm{PaO}_{2}\right)$ to fraction of inspiration $\mathrm{O}_{2}\left(\mathrm{FiO}_{2}\right)$. Other outcomes were muscle strength evaluation and visual analogue score (VAS) during rest and movement in 12 hours after surgery.

\section{Statistical Analysis}

SPSS 23.0 software (SPSS Inc, Chicago, IL) was used to process and analyze the data. Measurement data in line with normal distribution were expressed as Mean $\pm S D$, and tested by $t$ test. Measurement data not coincided with normal distribution were expressed as Median (Q1, Q3), and tested by Wilcoxon's Sign Rank Test. Enumeration data were expressed as percentage and tested by Fisher's Exact Test. A value of $P<0.05$ was considered statistically significant.

\section{Results}

A total of 93 patients undergoing shoulder arthroscopy were included between June and September 2019 and no patient was excluded. Demographic and surgical data were list in Table 1. The volume of fluid infusion was $831.67 \pm 224.96 \mathrm{ml}$, calculated by weight of the patients. 
Table 1

Demographic and Surgical Data

\begin{tabular}{|ll|}
\hline & Value \\
\hline Sex $(\mathrm{M} / \mathrm{F})$ & $45 / 48$ \\
\hline Age $(\mathrm{y})$ & $55.73 \pm 13.75$ \\
\hline $\mathrm{BMI}\left(\mathrm{kg} / \mathrm{m}^{2}\right)$ & $23.33 \pm 2.97$ \\
\hline Urine $(\mathrm{ml})$ & $310.00 \pm 167.84$ \\
\hline Bleeding $(\mathrm{ml})$ & $30.67 \pm 15.74$ \\
\hline Operation time (min) & $118.87 \pm 44.16$ \\
\hline Anesthesia time (min) & $183.16 \pm 50.94$ \\
\hline Fuid infusion volume (ml) & $831.67 \pm 224.96$ \\
\hline Irrigation (L) & $24.55 \pm 13.24$ \\
\hline Data are mean \pm SD & \\
\hline Abbreviation: $M$, male; $F$, female; BMl, body mass index. \\
\hline B lines (Fig. 1) failed to be detected before surgery, however, it appeared in most patients after \\
\hline surgery. According to $\mathrm{N}_{\mathrm{B}}$, patients were divided into 4 groups. \\
\hline
\end{tabular}

The highest total incidence of B lines was $49.4 \%$, occured at 4 hours after surgery. The highest incidence of moderate pulmonary edema was $9.7 \%(P=0.002)$. These incidences above were higher in comparison with that before operation $(P<0.05)$. The highest incidence of severe pulmonary edema was $3.2 \%$ and there was no significant difference when compared with that before operation. However, the value of $P$ was $0.081 \%$, which was too close to $0.05 \%$. (Table 2, Fig. 2) A total of three patients, unable to deoxygenate, were diagnosed with severe pulmonary edema and should be monitored in PACU. Furosemide was administrated, and all B lines disappeared at the time 4 hours after operation. All patients were sent back to the ward when the OI returned to the preoperative level after deoxygenation. 
Table 2

Incidence of B Lines at Different Time

\begin{tabular}{|c|c|c|c|c|c|c|}
\hline Level/Time & $T_{p a}(n=93)$ & $T_{2}(n=93)$ & $T_{4}(n=93)$ & $T_{6}(n=93)$ & $T_{8}(n=93)$ & $T_{10}(n=93)$ \\
\hline None & $18(19.7 \%)$ & $6(6.4 \%)$ & $13(13.9 \%)$ & $18(19.7 \%)$ & $27(29.0 \%)$ & $9(9.7 \%)$ \\
\hline Mild & $21(22.6 \%)$ & $27(29.0 \%)$ & $21(22.6 \%)$ & $6(6.5 \%)$ & $6(6.5 \%)$ & $3(3.2 \%)$ \\
\hline Moderate & 0 & 0 & $9(9.7 \%)$ & $6(6.5 \%)$ & 0 & 0 \\
\hline Severe & $3(3.2 \%)$ & $3(3.2 \%)$ & $3(3.2 \%)$ & 0 & 0 & 0 \\
\hline Total & $42(45.5 \%)$ & $36(38.6 \%)$ & $46(49.4 \%)$ & $30(32.7 \%)$ & 33 (35.5\%) & $12(12.9 \%)$ \\
\hline \multicolumn{7}{|c|}{ Data are number (\%) } \\
\hline $\begin{array}{l}\text { Abbreviation } \\
\text { time at } 4 \mathrm{hol} \\
\text { time at } 10 \mathrm{hc}\end{array}$ & $s$ after surge & (1) & end of cul & $; \mathrm{T}_{2}$, time & hours afte & $\begin{array}{l}\text { gery; } T_{4} \\
\text { gery; } T_{10}\end{array}$ \\
\hline
\end{tabular}

$B$ lines were found on both the affected side and the healthy side. There were no statistical difference in the number of $B$ lines on both sides at $T_{p a}, T_{2}, T_{8}, T_{10}$. The number of $B$ lines on the affected side was larger than that on the healthy side at $T_{4}, T_{6}$, and the difference was statistically significant.

Respiratory parameters including $\mathrm{OI}$ and arterial partial pressure of carbon dioxide $\left(\mathrm{PaCO}_{2}\right)$ were monitored before and during operation. The patients were divided into type 1 respiratory failure group (T1RF:Ol $<300 \mathrm{mmHg}$ and $\mathrm{PaCO}_{2}<50 \mathrm{mmHg}$ ) and type 2 respiratory failure group (T2RF:Ol $<300 \mathrm{mmHg}$ and $\mathrm{PaCO} 2>50 \mathrm{mmHg}$ ) by OI and $\mathrm{PaCO}_{2}$. For saving expenses, we failed to collect the correct breathing data after surgery, such as BGA. All the patients had normal respiratory parameters $(\mathrm{OI}>300 \mathrm{mmHg}$, $\mathrm{PaCO} 2<45 \mathrm{mmHg})$ before operation. However, during operation the incidence of T1RF was $5.4 \%(P=$ $0.023)$ and that of T1RF + T2RF were $6.5 \%(P=0.013)$. Both the difference above were significant compared with that before surgery $(P<0.05)$. (Table 3$)$

Table 3

Incidence of Respiratory Failure Before and During Operation

\begin{tabular}{|llll|}
\hline & Before Operation $(\mathbf{n}=\mathbf{9 3})$ & On Operation $(\mathbf{n}=\mathbf{9 3})$ & $\mathbf{P}$ \\
\hline T1RF & 0 & $5(5.4 \%)$ & 0.023 \\
\hline T2RF & 0 & $1(1.1 \%)$ & 0.316 \\
\hline T1RF + T2RF & 0 & $6(6.5 \%)$ & 0.013 \\
\hline Data are number (\%) & & \\
\hline Abbreviation: T1RF: type 1 respiratory failure group; T2RF: type 1 respiratory failure group. \\
\hline
\end{tabular}


VAS of resting and motion were $2.94 \pm 1.18$ and $5.26 \pm 1.81$ before nerve block. For the benefits from nerve block, pain was relieved in 6 hours after surgery (VAS $<3)$. At 12 hours after operation, the VAS of resting and motion were $4.68 \pm 2.27,6.90 \pm 2.43$, respectively. While the grade of muscle strength was $4.48 \pm 0.51$ at 12 hours after operation.

\section{Discussion}

The primary outcome was the number of $B$ lines in this study. The patients with pulmonary diseases (chronic pulmonary diseases or pulmonary edema) were not involved and B lines could be detected in none of the subjects before surgery. Although numerous minor and major atelectasis, which can also lead $B$ lines, are usual after ISBPB due to some phrenic nerve dysfunction [12], this probability could be excluded in this study for the $B$ lines were also located in health side rather than in the operation side only where atelectases were lead by nerve block.

Early evaluation is the key to the management and treatment of this complication in shoulder arthroscopy. Swan-Ganz catheter, PiCCO or Flowtrac/Vigileo system, as the direct and real-time tools for monitoring circulation overload, were not available to monitor patients for the predictable trauma and high expense. Multiple studies have shown $\mathrm{N}_{B}$ could be an indirect index to reflect the circulatory overload $[12,13]$. The sensitivity and specificity of $B$ lines for the diagnosis of extracascular water (EVLW $>500 \mathrm{ml}$ ) were about $90 \%$ and $86 \%$, respectively. While the sensitivity and specificity are also high to detect EVLW below $500 \mathrm{ml}$, indicating ultrasound can be beneficial for early detection of circulation overload [14]. $N_{B}$ in this study to evaluate the severity of pulmonary edema was based on the international experts consensus of pulmonary ultrasound in 2012 [11]. Studies have shown that $N_{B}$ changed with the volume of EVLW, which also indicated $N_{B}$ could reflecte the severity of pulmonary edema [15]. The advantages to detect $B$ lines by ultrasound are very prominent. First of all, the most obvious feature is convenient and easy. In terms of counting $\mathrm{N}_{\mathrm{B}}$, with a portable ultrasonic instrument, the beginner can achieve the same effect after just 1 hour of training as the top ultrasonic instrument used by experienced experts [16]. Second, ultrasound also has great advantages of safety, which is radiation-free [17].

Despite the high incidence of this complication, the methods below can help improve patients' safety in shoulder arthroscopy. In this study, all of the patients were under operation in the lateral decubitus position, which could increase the risk of extravasation and lead the airway compromise for the gravity [18]. Borgeat and Hynson reported two cases of airway compromise in the lateral decubitus position [7]. In this position, the brachial plexus will also be more likely to be injuried for continuous tractive effort [19]. The beach-chair position, requiring less irrigation, has many advantages recommanded by large number of studies. 1.More visual field: the surgeon will be allowed to easily judge the anatomic structures [20]; 2.A wider range of intraoperative shoulder activity: the shoulder joint will be rotated easily for the lack of traction [21]; 3. Less bleeding: it provides a lower blood pressure and a better exposure; 4 . More convenient for switching to open surgery: when shoulder arthroscopy is not successful, it can be converted to open surgery directly under this position [22]. 
The irrigation under this surgery is mainly perfused by gravity or automatic pressure device [23]. However, in order to limit the high pressure by the device, irrigation pump powered by gravity is recommended [24]. All the irrigation water were placed on the same level and pumped by gravity in this study.

Isosmotic saline is commonly used in shoulder arthroscopy. However, it may lead a large amount of extravasation into the periarticular tissue space during long time surgery $[6,25]$. Lo reported the weight gain of patients would increase to $3.95 \pm 1.77 \mathrm{~kg}$ after surgery [26]. Therefore, the isosmotic saline in this study might increase the risk of fluid extravasation. Hypertonic solution may be a better choice for this surgery. A study reported the weight gain of patients with hypertonic irrigation was $2.25 \pm 0.77 \mathrm{~kg}$ and the use of hypertonic lavage did not increase operating time [27]. Studies also have shown isotonic or hypotonic extracellular fluid might aggravate the death of chondrocytes in articular cartilage [28]. In contrast, hypertonic lavage fluid had a protective effect on articular cartilage [29]. Animal experiments showed hypertonic fluid could reduce chondrocyte damage and enhance cartilage repairment [30]. Hypertonic solution could not only reduce fluid exudation effectively, but was also beneficial to the recovery of cartilage.

Currently, studies had proved that patients with amount of irrigation less than $20 \mathrm{~L}$ were safe. While in patients with symptoms, the total volume of fluid usually ranged from 20 to $36 \mathrm{~L}$ [31]. That was about $24.55 \pm 13.24 \mathrm{~L}$ in this study, which could be a risk leading pulmonary edema. As a result, it is an important way to decrease the total amount of lavage fluid. In order to limit the volume and improve surgical view, surgeons may consider using electrocautery devices, epinephrine-infused fluids and proper controlled hypotension [32]. However, prolonged surgery may also increase total volume of perfusion. Studies showed the duration of this surgery should be less than $120 \mathrm{~min}$ [24]. The critical value of operative time to lead airway compromise is about $150 \mathrm{~min}[33]$. As a result, risk can be reduced by decreasing the operation time.

Anesthesiologists can also reduce the incidence of complications. At present, general anesthesia combined with ISBPB is recommended for shoulder arthroscopy. 1.Better control of respiration and circulation: endotracheal intubation can provides a secure airway; controlled hypotension can effectively reduce bleeding[34]. 2.Lower incidence of complications with general anesthesia: general anesthesia is a safe technology, and the mortality related with anaesthesia is only 1 in 300,000. 3.Better management of patients: since the shoulder joint is close to the head, local anesthesia alone may make patients nervous, and the surgical drapes can also cause claustrophobia or other discomfort[35]. General anesthesia can eliminate psychological factors from the patients. The benefits of brachial plexus block include not only excellent muscle relaxation, reduction of opioids but the adequate postoperative analgesia[36]. Therefore, single general anesthesia is not recommended in shoulder arthroscopy [37].

It is also important for timely recognize the change of airway pressure and lung compliance [[38]]. The tracheal tube cuff deflating test is very helpful to identify edema around the airway. The cuff is deflated in the machine-control breathing mode, and the tidal volume (TV) is recorded. There is leakage around the tracheal tube when TV decreases by more than $100 \mathrm{ml}$ (positive deflating test). If the test is positive, 
muscle relaxant antagonist is administrated after spontaneous breathing and the tracheal tube can be removed. If there is no leakage around the endotracheal tube (negative deflating test), indicating edema around the airway and extubation is not recommended.

The limitation is that this is an observational study without any intervention, such as the use of fursemi, hormones, or positive end-expiratory pressure ventilation (PEEP). Central venous catheters were not performed, which could be used for the evaluation of blood volume. Future randomized controlled trials also need to be conducted to study interventions to reduce pulmonary edema.

In conclusion, pulmonary edema should't be neglected, for there is a high incidence of this complication in shoulder arthroscopy, which may affect air exchange to decrease oxygenation capacity. The ultrasound is an convenient tool to rapidly assess and identify this complication. Pain can be relieved in 6 hours after surgery by ISBPB with ropivacaine and muscle strength can also recover at 12 hours after surgery.

\section{Abbreviations}

ISBPB

Interscalene brachial plexus block; PACU:Postanesthesia care unit; RW:Remaining weight; BGA:Blood gas analysis, Ol:Oxygenation index; PaO2:Partial arterial 02 pressure; PaCO2:Partial arterial $\mathrm{CO} 2$ pressure; FiO2:Fraction of inspiration 02; VAS:Visual analogue score; T1RF:Type 1 respiratory failure; T2RF:Type 2 respiratory failure; EVLW:Extracascular water; PEEP:end-expiratory pressure ventilation.

\section{Declarations}

\section{Acknowledgements}

We would like to thank Dr. Si Zeng and Dr. Su Li for the assistance with the study.

\section{Authors' contributions}

GZ designed the study, conducted the study, analyzed the data and wrote the manuscript. QHW designed the study, conducted the study, analyzed the data and wrote the manuscript. XYH conducted the study, analyzed the data and wrote the manuscript. YHS and CQL helped design the study. LZ helped design the study, analyze the data and revise the manuscript. All authors read and approved the final manuscript.

\section{Funding}


Supported by Sichuan Science and Technology Program entitled "Study on clinical pathway of anesthesia to shoulder arthroscopic " (20ZDYF1325). The funders are Dr. Su Li, Dr. Yunhua Shui, Dr Chunqiong Luo.

\section{Availability of data and materials}

The datasets used and/or analysed during the current study are available from the corresponding author on reasonable request.

\section{Ethics approval and consent to participate}

This observational trial was conducted from June 2019 to September 2019 at Sichuan Provincial Orthopedic Hospital (Chengdu Sports Hospital and Chengdu Research Institute for Sports Injury), Chengdu, China Ethical approval for this study was provided by the Ethical Committee of Sichuan Provincial Orthopedic Hospital (Chairperson Prof Hai Shen, Number: 2019sgkl-004-01) on 4 June 2019. The written informed consents were obtained from all patients before participation.

\section{Consent for publication}

The authors declare that they have consented it to be published in BMC Anesthesiology.

\section{Competing interests}

The authors declare that they have no competing interests.

\section{Author details}

1Department of Anesthesia, Sichuan Provincial Orthopedic Hospital (Chengdu Sports Hospital and Chengdu Research Institute for Sports Injury), Chengdu, China

2Chengdu University of Traditional Chinese Medicine, Chengdu, China

3Department of Operation Room, the Third People's Hospital of Chengdu, Chengdu, China.

*: Equal contributor. 


\section{References}

1.

Antonucci S,., Orlandi P, Mattei. PA, et al. Airway obstruction during arthroscopic shoulder surgery: anesthesia for the patient or for the surgeon? Minerva Anestesiol. 2006;72(12):995-1000.

2.

Memon M, Kay J, Gholami A, et al. Fluid Extravasation in Shoulder Arthroscopic Surgery: A Systematic Review. Orthop J Sports Med. 2018;6(5):2325967118771616.

3.

Venkat G, Moon YL, Na WC, et al. Upper airway compromise by extravasated fluid: a rare complication after arthroscopic repair of atrophic cuff tear. Orthopedics. 2009;32(10).

4.

Jirativanont T.., Tritrakarn TD. Upper airway obstruction following arthroscopic rotator cuff repair due to excess irrigation fluid. Anaesthesia Intensive Care. 2010;38(5):957-8.

5 .

Anoop Raj G, Jeetendra B, Ameeta S, et al. Negative-pressure pulmonary oedema in a patient undergoing shoulder arthroscopy. Indian Journal of Anaesthesia. 2012;56(1):62-5.

6.

Bhaskar SB, Manjuladevi M. Shoulder arthroscopy and complications: Can we afford to relax? Indian Journal of Anaesthesia. 2015;59(6):335-7.

7.

Borgeat A, Bird P, Ekatodramis $G$, et al. Tracheal compression caused by periarticular fluid accumulation: A rare complication of shoulder surgery *. Journal of Shoulder Elbow Surgery. 2000;9(5):443-5.

8.

Wang JH, He Q,., Liu YL, et al. Pulmonary edema in the transurethral resection syndrome induced with mannitol 5\%. Acta Anaesthesiol Scand. 2010;53(9):1094-6.

9.

Ming-Hui $\mathrm{H}$, Ta-Liang $\mathrm{C}$, Yu-Hua L, et al. Acute pulmonary edema from unrecognized high irrigation pressure in hysteroscopy: a report of two cases. J Clin Anesth. 2008;20(8):614-7. 10.

$\mathrm{Ma} \mathrm{H}$, Huang $\mathrm{D}$, Zhang $\mathrm{M}$, et al. Lung ultrasound is a reliable method for evaluating extravascular lung water volume in rodents. Bmc Anesthesiology. 2015;15(1):162.

11.

Picano E, Frassi F, Agricola E, et al. Ultrasound Lung Comets: A Clinically Useful Sign of Extravascular Lung Water. J Am Soc Echocardiogr. 2006;19(3):356-63.

12.

Dietrich CF, Mathis G, Blaivas M, et al. Lung B-line artefacts and their use. Journal of Thoracic Disease. 2016;8(6):1356-65.

13.

Lichtenstein DA. Lung Ultrasound in the Critically III. 2005;16(2):79-87. 
14.

Eustachio A, Tiziana B, Michele O, et al. "Ultrasound comet-tail images": a marker of pulmonary edema: a comparative study with wedge pressure and extravascular lung water. Chest. 2005;127(5):1690-5. 15.

Gino S, Valeria G, Sara S, et al. "Synthetic" comets: a new look at lung sonography. Ultrasound in Medicine Biology. 2011;37(11):1762-70.

16.

Chiem AT, Chan CH, Ander DS, et al. Comparison of Expert and Novice Sonographers' Performance in Focused Lung Ultrasonography in Dyspnea (FLUID) to Diagnose Patients With Acute Heart Failure Syndrome. Acad Emerg Med. 2015;22(5):564-73.

17.

Eugenio P, Eliseo V, Rehani MM, et al. The appropriate and justified use of medical radiation in cardiovascular imaging: a position document of the ESC Associations of Cardiovascular Imaging, Percutaneous Cardiovascular Interventions and Electrophysiology. Eur Heart J. 2014;35(10):665-72. 18.

Hynson JM, Tung A,., Guevara JE, et al. Complete airway obstruction during arthroscopic shoulder surgery. Anesthesia Analgesia. 1993;76(4):875-8.

19.

Rains DD, Alec G, Wahl R. CJ. Pathomechanisms and complications related to patient positioning and anesthesia during shoulder arthroscopy. Arthroscopy the Journal of Arthroscopic Related Surgery. $2011 ; 27(4): 532-41$.

20.

Provencher CMT, Mcintire ES, Gaston TM, et al. Avoiding Complications in Shoulder Arthroscopy: Pearls for Lateral Decubitus and Beach Chair Positioning. Tech Should Elbow Surg. 2010;11(1):1-3. 21.

Terry MA, Altchek DW. Diagnostic Shoulder Arthroscopy Technique: Beach Chair Position. 2003. 22.

Mannava S, Jinnah AH, Plate JF, et al. Basic Shoulder Arthroscopy: Beach Chair Patient Positioning. Arthroscopy Techniques. 2016;5(4):e731-5.

23.

Montfoort DOV, Huijsmans PE. Epinephrine Diluted Saline-Irrigation Fluid in Arthroscopic Shoulder Surgery: A Significant Improvement of Clarity of Visual Field and Shortening of Total Operation Time. Arthroscopy the Journal of Arthroscopic Related Surgery. 2013;29(10):e88-9.

24.

Ferhan K, Sampathila P, Manjula S, et al. Airway compromise due to irrigation fluid extravasation following shoulder arthroscopy. Journal of Anaesthesiology Clinical Pharmacology. 2013;29(4):578-9. 25.

Gupta S, Manjuladevi M, Upadhyaya KV, et al. Effects of irrigation fluid in shoulder arthroscopy. Indian Journal of Anaesthesia. 2016;60(3):194-8.

26. 
Lo IKY, Burkhart SS. Immediate postoperative fluid retention and weight gain after shoulder arthroscopy. Arthroscopy-the Journal of Arthroscopic Related Surgery. 2005;21(5):605-10.

27.

Yang M, Han Y, Zong L, et al. Evaluation of hypertonic lavage' safety and efficacy in shoulder arthroscopy.

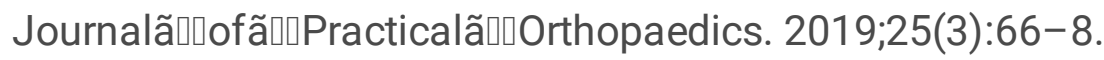

28.

Huang Y, Zhang Y, Ding X, et al. Osmolarity influences chondrocyte repair after injury in human articular cartilage. Journal of Orthopaedic Surgery Research. 2015;10(1):1-9. ,10,1(2015-01-28).

29.

Amin AK, Huntley JS, Simpson AHRW, et al. Increasing the Osmolarity of Joint Irrigation Solutions May Avoid Injury to Cartilage: A Pilot Study. Clinical Orthopaedics Related Research®. 2010;468(3):875-84. 30 .

Eltawil NM, Howie SEM, Simpson AHRW, et al. The use of hyperosmotic saline for chondroprotection: implications for orthopaedic surgery and cartilage repair. Osteoarthritis Cartilage. 2015;23(3):469-77. 31.

Ercin E, Bilgili MG, Ones HN, et al. Postoperative pectoral swelling after shoulder arthroscopy. Joints. 2015;03(03):158-60.

32.

Jensen $\mathrm{KH}$, Werther $\mathrm{K}_{\text {,., }}$ Stryger $\mathrm{V}$, ., et al. Arthroscopic shoulder surgery with epinephrine saline irrigation. Arthroscopy the Journal of Arthroscopic Related Surgery. 2001;17(6):578-81.

33.

Edwards DS, Davis I, Jones NA, et al. Rapid tracheal deviation and airway compromise due to fluid extravasation during shoulder arthroscopy. Journal of Shoulder Elbow Surgery. 2014;23(7):e163-5.

34.

Gillespie R, Shishani Y, Streit J, et al. The safety of controlled hypotension for shoulder arthroscopy in the beach-chair position. Journal of Bone Joint Surgery American Volume. 2012;94(14):1284-90. 35 .

Dove P, Gilmour F, Weightman WM, et al. Patient perceptions of regional anesthesia: influence of gender, recent anesthesia experience, and perioperative concerns. Regional Anesthesia Pain Medicine. 2011;36(4):332.

36.

Stephan B, Marco N, Christian G, et al. Severe airway obstruction during arthroscopic shoulder surgery. Anesthesiology. 2003;99(6):1455-6.

37.

Wu CL, Rouse LM, Chen JM, et al. Comparison of postoperative pain in patients receiving interscalene block or general anesthesia for shoulder surgery. Orthopedics. 2002;25(1):45-8.

38.

Manjuladevi M,., Surbhi G, Ks Vasudeva U, et al. Postoperative airway compromise in shoulder arthroscopy: A case series. Indian Journal of Anaesthesia. 2013;57(1):52-5. 


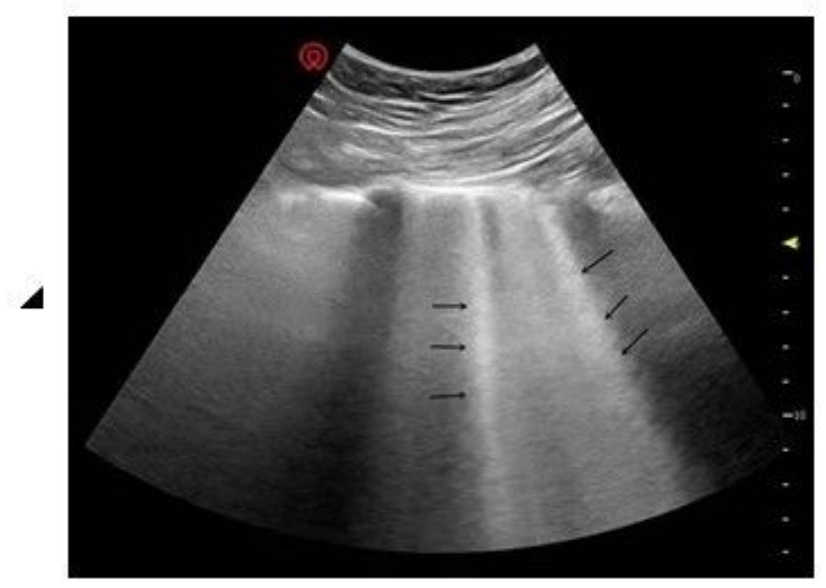

\section{Figure 1}

B lines were detected by ultrasound (black arrows)

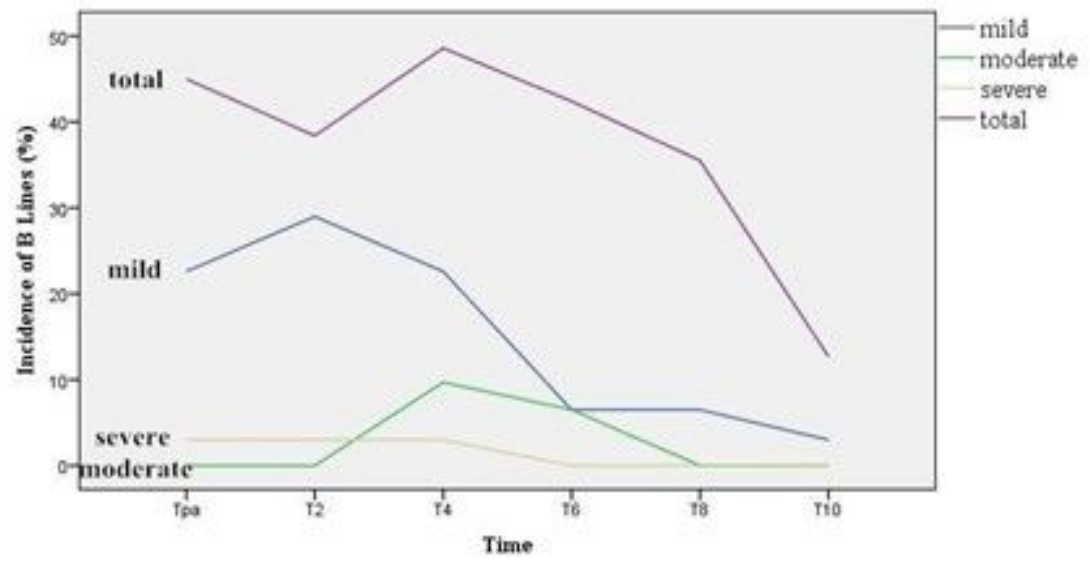

Figure 2

The curves indicated incidence of B lines from the end of surgery to 10 hours after operation. Abbreviation: Tpa, time in PACU (or time at the end of surgery); T2, time at 2 hours after surgery; T4, time at 4 hours after surgery; T6, time at 6 hours after surgery; T8, time at 8 hours after surgery; T10, time at 10 hours after surgery. 